\title{
Full Scale Nonlinear Electromagnetic Inversion for Biological Objects
}

\author{
Aria Abubakar and Peter M. van den Berg \\ Centre for Technical Geosciences, Delft University of Technology \\ Mekelweg 4, 2628 CD, Delft, The Netherlands \\ abubakar@its.tudelft.nl
}

\begin{abstract}
In this paper the contrast source inversion method using a multiplicative weighted $L^{2}$-norm total variation regularizer is applied to two- and three-dimensional image reconstructions from electromagnetic microwave tomography experiments. This iterative method avoids solving a full forward problem in each iteration which makes the method suitable to handle a large scale computational problem. The numerical results both from simulation and experimental data with high contrast biological phantom are presented and discussed.
\end{abstract}

\section{Introduction}

Image reconstruction is a complicated nonlinear problem in microwave tomography because both the material parameters and the field distribution in the investigation domain are unknowns. Serious efforts have been made to solve this problem in two-dimensional model where the scalar Helmholtz equation (TM electromagnetic polarization) can be applied. If the unknown object has small contrast of dielectric properties, a linearization procedure like the Born approximations [1] can be used to provide images with spatial resolution of a fraction of the wavelength. Computer codes based on these approximations demonstrate a high speed and can be used for almost real time imaging. Iterative procedures for the Born approximation have been used for intermediate contrast objects [4].

More complicated mathematical reconstruction algorithms have been developed for reconstruction objects with high contrast in dielectric properties [3, $5-8,10]$. These algorithms require large computer resources, but nevertheless theoretically provide a resolution with a sufficiently high signal-to-noise ratio. The Newton method [11] has been successfully applied to high contrast objects. The main bottlenecks of this Newton approach, especially discouraging in the three-dimensional case, are the multiple forward solutions needed to construct the Hessian matrix. Another type of method which avoids solving any forward problem in each iterative step is introduced in $[9,12]$. This approach can solve the problem without dealing with a high-dimensional linear equation system but it requires a larger number of iterations.

Recently, this type of method has been armed with a total variation regularizer in order to handle high contrast objects [13]. Although the addition of 
the total variation to the cost functional has a very positive effect on the quality of the reconstructions for both 'blocky' and smooth profiles, a drawback is the presence of an artificial weighting parameter in the cost functional, which can only be determined through considerable numerical experimentation and a priori information of the desired reconstruction. In [13], it was suggested to include the total variation as a multiplicative constraint, with the result that the original cost functional is the weighting parameter of the regularizer, so that this parameter is determined by the inversion procedure itself. This eliminates the choice of the artificial regularization parameters completely. The multiplicative type of regularization seems to handle noisy as well as limited data in a robust way without the usually necessary a priori information. In this paper the latter method using the new weighted $L^{2}$-norm total variation regularizer introduced in [14] is applied to handle more complicated biological objects embedded in a lossy medium. Numerical examples using simulation and experimental data demonstrate the ability of the presented method.

\section{Problem Statement}

We consider an object, $B$, of arbitrary bounded cross section with complex dielectric permittivity $\varepsilon(\boldsymbol{x})$ and arbitrary shape. The complex permittivity and the shape of this object $B$ are unknown, but they are known to lie within a bounded simply connected object domain $D$. This object domain $D$ is assumed to be embedded in the background medium, the immersion liquid, with permittivity $\varepsilon_{\mathrm{b}}$. The position vector is denoted by $\boldsymbol{x}$. We assume a time harmonic dependence $\exp (\mathrm{j} \omega t)$, where $\mathrm{j}^{2}=-1, \omega$ is angular frequency, and $t$ is time. We also assume that the object is irradiated successively by a number of known incident electric fields $\boldsymbol{E}_{s}^{\text {inc }}(\boldsymbol{x})=\boldsymbol{E}^{\mathrm{inc}}\left(\boldsymbol{x}, \boldsymbol{x}_{s}^{\mathrm{S}}\right), s=1, \cdots$, originating from source positions $\boldsymbol{x}_{s}^{\mathrm{S}}$.

For each incident field, the total electric field will be denoted by $\boldsymbol{E}_{s}$. Nowadays, it is well-known that the total field $\boldsymbol{E}_{s}$ and the scattered field $\boldsymbol{E}_{s}^{\text {sct }}$ satisfy the following domain integral representations

$$
\begin{aligned}
\boldsymbol{E}_{s}(\boldsymbol{x}) & =\boldsymbol{E}_{s}^{\mathrm{inc}}(\boldsymbol{x})+\left(k_{\mathrm{b}}^{2}+\boldsymbol{\nabla} \boldsymbol{\nabla} \cdot\right) \int_{D} g\left(\boldsymbol{x}-\boldsymbol{x}^{\prime}\right) \chi\left(\boldsymbol{x}^{\prime}\right) \boldsymbol{E}_{s}\left(\boldsymbol{x}^{\prime}\right) \mathrm{d} v\left(\boldsymbol{x}^{\prime}\right), \quad \boldsymbol{x} \in D, \\
\boldsymbol{E}_{s}^{\mathrm{sct}}(\boldsymbol{x}) & =\left(k_{\mathrm{b}}^{2}+\boldsymbol{\nabla} \boldsymbol{\nabla} \cdot\right) \int_{D} g\left(\boldsymbol{x}-\boldsymbol{x}^{\prime}\right) \chi\left(\boldsymbol{x}^{\prime}\right) \boldsymbol{E}_{s}\left(\boldsymbol{x}^{\prime}\right) \mathrm{d} v\left(\boldsymbol{x}^{\prime}\right), \quad \boldsymbol{x} \notin D,
\end{aligned}
$$

where $\chi(\boldsymbol{x})=\varepsilon(\boldsymbol{x}) / \varepsilon_{\mathrm{b}}-1$ is the contrast, $g(\boldsymbol{x})=\exp \left(-\mathrm{j} k_{\mathrm{b}}|\boldsymbol{x}|\right) / 4 \pi|\boldsymbol{x}|$ is the Green's function, and $k_{\mathrm{b}}=\omega\left(\varepsilon_{\mathrm{b}} \mu_{0}\right)^{1 / 2}$ denotes the wavenumber in the embedding.

In the inverse scattering problem $\boldsymbol{F}_{s}=\boldsymbol{E}_{s}^{\text {sct }}$ will be measured on some domain $S$ outside $D$, so the integral representation in (2) for points exterior to $D$ is written symbolically as the data equation,

$$
\boldsymbol{F}_{s}=\mathcal{G}^{S} \chi \boldsymbol{E}_{s}, \quad \boldsymbol{x} \in S,
$$

while the integral equation in (1) is written symbolically as the object equation,

$$
\boldsymbol{E}_{s}^{\text {inc }}=\boldsymbol{E}_{s}-\mathcal{G}^{D} \chi \boldsymbol{E}_{s}, \quad \boldsymbol{x} \in D,
$$


where the operator $\mathcal{G}^{S}$ is an operator mapping from $L^{2}(D)$ into $L^{2}(S)$ and the operator $\mathcal{G}^{D}$ is an operator mapping $L^{2}(D)$ into itself.

The inverse scattering problem consists of determining $\chi$ from a knowledge of the incident fields, $\boldsymbol{E}_{s}^{\text {inc }}$, on $D$ and the scattered fields, $\boldsymbol{F}_{s}$, on $S$. Because the total fields $\boldsymbol{E}_{s}$ on $D$ are also unknown, this problem is non-linear. In practice it can only be solved iteratively due to a large number of unknowns.

\section{Inversion Algorithm}

In this method, one chooses to reconstruct the material contrast $\chi$ and the contrast sources $\boldsymbol{W}_{s}$ instead of the fields $\boldsymbol{E}_{s}$. The contrast sources are defined by

$$
\boldsymbol{W}_{s}(\boldsymbol{x})=\chi(\boldsymbol{x}) \boldsymbol{E}_{s}(\boldsymbol{x}) .
$$

Using (5) in (3), the data equation becomes

$$
\boldsymbol{F}_{s}=\mathcal{G}^{S} \boldsymbol{W}_{s}, \quad \boldsymbol{x} \in S
$$

while the object equation becomes

$$
\boldsymbol{E}_{s}=\boldsymbol{E}_{s}^{\mathrm{inc}}+\mathcal{G}^{D} \boldsymbol{W}_{s}, \quad \boldsymbol{x} \in D
$$

Substituting (7) into (5), we obtain an object equation for $\boldsymbol{W}_{s}$

$$
\chi \boldsymbol{E}_{s}^{\text {inc }}=\boldsymbol{W}_{s}-\chi \mathcal{G}^{D} \boldsymbol{W}_{s}, \quad \boldsymbol{x} \in D .
$$

Then, in the Contrast Source Inversion (CSI) method [12] using the multiplicative weighted $L^{2}$-norm Total Variation (TV) regularization factor [14] (denoted by MR-CSI), the sequences of $\boldsymbol{W}_{s, n}$ and $\chi_{n}$ for $n=1,2, \cdots$, are iteratively found by minimizing a cost functional, viz.,

$$
\begin{aligned}
& F_{n}\left(\boldsymbol{W}_{s}, \chi\right)=\left[F^{S}\left(\boldsymbol{W}_{s}\right)+F_{n}^{D}\left(\boldsymbol{W}_{s}, \chi\right)\right] F_{n}^{R}(\chi), \\
& \quad=\left[\eta^{S} \sum_{s}\left\|\boldsymbol{F}_{s}-\mathcal{G}^{S} \boldsymbol{W}_{s}\right\|_{S}^{2}+\eta_{n}^{D} \sum_{s}\left\|\chi \boldsymbol{E}_{s}^{\text {inc }}-\boldsymbol{W}_{s}+\chi \mathcal{G}^{D} \boldsymbol{W}_{s}\right\|_{D}^{2}\right] F_{n}^{R}(\chi),
\end{aligned}
$$

where $F^{S}$ and $F_{n}^{D}$ are the errors in the data and object equations. The normalization factors in the cost functional are chosen as $\eta^{S}=\sum_{s}\left\|\boldsymbol{F}_{s}\right\|_{S}^{2}$ and $\eta_{n}^{D}=\sum_{s}\left\|\widetilde{\chi} \boldsymbol{E}_{s}^{\text {inc }}\right\|_{D}^{2}$, respectively. Further, $\|\cdot\|_{S, D}^{2}$ denotes the squared norm on $S / D$. The weighted $L^{2}$-norm TV factor is as introduced in [14],

$$
F_{n}^{R}(\chi)=\frac{1}{\int_{D} \mathrm{~d} v(\boldsymbol{x})} \int_{D} \frac{|\boldsymbol{\nabla} \chi|^{2}+\delta_{n}^{2}}{|\boldsymbol{\nabla} \widetilde{\chi}|^{2}+\delta_{n}^{2}} \mathrm{~d} v(\boldsymbol{x})=\left\|b_{n} \boldsymbol{\nabla} \chi\right\|_{D}^{2}+\delta_{n}^{2}\left\|b_{n}\right\|_{D}^{2},
$$

where $b_{n}=\left[\int_{D} \mathrm{~d} v(\boldsymbol{x})\left(|\boldsymbol{\nabla} \tilde{\chi}|^{2}+\delta_{n}^{2}\right)\right]^{-1 / 2}$. In (9) and (10) $\tilde{\chi}$ is some particular contrast value. Its choice will be determined later. Although the constant parameter $\delta_{n}^{2}$ is introduced for restoring differentiability of the regularizer, it also controls the influence of the regularization. We therefore have chosen to increase 
the regularization as a function of the number of iterations by decreasing this parameter $\delta_{n}^{2}$. Since the normalized object error term will decrease as a function of the number of iterations, we choose

$$
\delta_{n}^{2}=F_{n}^{D}\left(\boldsymbol{W}_{s, n}, \widetilde{\chi}\right) \tilde{\Delta}^{2},
$$

where $\tilde{\Delta}$ denotes the reciprocal mesh size of the discretized domain $D$. Its choice is inspired by the idea that in the first few iterations, we do not need the minimization of the regularizer and as the iterations proceed we want to increase the effect of the regularizer.

The structure of the cost functional in (9) is such that it will minimize the factor $F_{n}^{R}$ with a large weighting parameter in the beginning of the optimization process, because the value of $F^{S}+F_{n}^{D}$ is still large, and that it will gradually minimize more and more the normalized errors in the data and object equations when the factor $F_{n}^{R}$ remains a nearly constant value close to one. If noise is present in the data, the term $F^{S}$ will remain at a large value during the whole optimization and therefore, the weight of the factor $F_{n}^{R}$ will be more significant. Hence, the noise will, at all times, be suppressed in the reconstruction process.

This MR-CSI method starts with back propagation as the initial estimates for the contrast sources and the contrast [12]. Then, in each iteration we first update the contrast sources $\boldsymbol{W}_{s, n}$ using a conjugate gradient step

$$
\boldsymbol{W}_{s}=\boldsymbol{W}_{s, n-1}+\alpha_{n} \boldsymbol{w}_{s, n},
$$

where the functions $\boldsymbol{w}_{s, n}$ are the Polak-Ribière conjugate gradient directions,

$$
\boldsymbol{w}_{s, 0}=\mathbf{0}, \quad \boldsymbol{w}_{s, n}=\partial \boldsymbol{w}_{s, n}+\frac{\sum_{s} \operatorname{Re}\left\langle\partial \boldsymbol{w}_{s, n}, \partial \boldsymbol{w}_{s, n}-\partial \boldsymbol{w}_{s, n-1}\right\rangle_{D}}{\sum_{s}\left\|\partial \boldsymbol{w}_{s, n-1}\right\|_{D}^{2}} \boldsymbol{w}_{s, n-1} .
$$

The gradient $\partial \boldsymbol{w}_{s, n}$ of the cost functional $F_{n}\left(\boldsymbol{W}_{s}, \chi\right)$ in (9) with respect to $\boldsymbol{W}_{s}$ evaluated at $\boldsymbol{W}_{s, n-1}$ and $\chi_{n-1}$ is given by

$$
\partial \boldsymbol{w}_{s, n}=-\eta^{S} \mathcal{G}^{S *} \boldsymbol{\rho}_{s, n-1}-\eta_{n}^{D}\left(\boldsymbol{r}_{s, n-1}-\mathcal{G}^{D *} \overline{\chi_{n-1}} \boldsymbol{r}_{s, n-1}\right),
$$

where $\mathcal{G}^{S *}$ and $\mathcal{G}^{D *}$ are the adjoint operators of $\mathcal{G}^{S}$ and $\mathcal{G}^{D}$, respectively, and the overbar denotes the complex conjugate. Further, the residuals are defined as

$$
\boldsymbol{\rho}_{s, n}=\boldsymbol{F}-\mathcal{G}^{S} \boldsymbol{W}_{s, n} \quad \text { and } \quad \boldsymbol{r}_{s, n}=\chi_{n} \boldsymbol{E}_{s}^{\mathrm{inc}}-\boldsymbol{W}_{s, n}+\chi_{n} \mathcal{G}^{D} \boldsymbol{W}_{s, n} .
$$

The real parameter $\alpha_{n}$ is found as minimizer of $F_{n}\left(\boldsymbol{W}_{s, n-1}+\alpha \boldsymbol{w}_{s, n}, \chi_{n-1}\right)$,

$$
\alpha_{n}=\frac{-\sum_{s} \operatorname{Re}\left\langle\partial \boldsymbol{w}_{s, n}, \boldsymbol{w}_{s, n}\right\rangle_{D}}{\eta^{S} \sum_{s}\left\|\mathcal{G}^{S} \boldsymbol{w}_{s, n}\right\|_{S}^{2}+\eta_{n}^{D} \sum_{s}\left\|\boldsymbol{w}_{s, n}-\chi_{n-1} \mathcal{G}^{D} \boldsymbol{w}_{s, n}\right\|_{D}^{2}} .
$$

Note that the cost functional in (9) is a quadratic function of $\alpha$, and the minimizer is unique. In the updating scheme for the contrast sources $\tilde{\chi}$ is chosen to be the contrast in the previous iteration, $\widetilde{\chi}=\chi_{n-1}$. Then $F_{n}^{R}\left(\chi_{n-1}\right)$ is always 
equal to one during the updating of $\boldsymbol{W}_{s, n}$. Subsequently, we compute the field $\boldsymbol{E}_{s, n}$ by substituting (12) in (4).

After $\boldsymbol{W}_{s, n}$ have been obtained, in each iteration, we proceed with updating of $\chi_{n}$. First, we observed that the closed-form expression of the contrast can be found if the regularization factor $F_{n}^{R}$ is absent, viz.,

$$
\chi_{n}=\arg \min \operatorname{complex}_{\chi}\left\{F^{S}\left(\boldsymbol{W}_{s, n}\right)+F_{n}^{D}\left(\boldsymbol{W}_{s, n}, \chi\right)\right\}=\frac{\sum_{s} \boldsymbol{W}_{s, n} \cdot \overline{\boldsymbol{E}_{s, n}}}{\sum_{s}\left|\boldsymbol{E}_{s, n}\right|^{2}} .
$$

From this point we make an additional minimization step,

$$
\chi_{n}^{R}=\chi_{n}+\beta_{n} d_{n},
$$

where $\chi_{n}$ is now given by (17) and $d_{n}$ is the Polak-Ribière conjugate gradient

$$
d_{0}=0, \quad d_{n}=g_{n}^{R}+\frac{\operatorname{Re}\left\langle g_{n}^{R}, g_{n}^{R}-g_{n-1}^{R}\right\rangle_{D}}{\left\|g_{n-1}^{R}\right\|_{D}^{2}} d_{n-1} .
$$

We remark that we prefer now a line minimization around the minimum of the cost functional $F^{S}+F_{n}^{D}$ (physical cost criterion). Then, during the updating of the contrast $\tilde{\chi}$ is taken to be equal to $\chi_{n}$ in (17). In view of (17) we take $g_{n}^{R}$ as

$$
\begin{aligned}
g_{n}^{R} & =\frac{\left[\frac{\partial F_{n}^{D}\left(\boldsymbol{W}_{s, n}, \chi\right)}{\partial \chi} F_{n}^{R}(\chi)+\left[F^{S}\left(\boldsymbol{W}_{s, n}\right)+F_{n}^{D}\left(\boldsymbol{W}_{s, n}, \chi\right)\right] \frac{\partial F_{n}^{R}(\chi)}{\partial \chi}\right]_{\chi=\chi_{n}}}{\sum_{s}\left|\boldsymbol{E}_{s, n}\right|^{2}} \\
& =\left[F^{S}\left(\boldsymbol{W}_{s, n}\right)+F_{n}^{D}\left(\boldsymbol{W}_{s, n}, \chi_{n}\right)\right] \frac{\boldsymbol{\nabla} \cdot\left(b_{n}^{2} \boldsymbol{\nabla} \chi_{n}\right)}{\sum_{s}\left|\boldsymbol{E}_{s, n}\right|^{2}},
\end{aligned}
$$

a preconditioned gradient of the cost functional $F_{n}\left(\boldsymbol{W}_{s, n}, \chi\right)$ with respect to changes in the contrast around the point $\chi=\chi_{n}$. In view of the previous minimization step, the gradient of $F_{n}^{D}$ with respect to changes in the contrast around the point $\chi=\chi_{n}$ vanishes. Hence, the gradient with respect to the contrast, in contrary to the previous approaches of the MR-CSI method [13,14], contains only a contribution of the additionally imposed regularization.

The real parameter $\beta_{n}$ is found from a line minimization as a minimizer of the cost functional in (9). The minimization of $F_{n}\left(\boldsymbol{W}_{s, n}, \chi_{n}+\beta d_{n}\right)$, which is a fourth-degree polynomial in $\beta$, can be performed analytically viz.,

$$
F_{n}=\left(A+B \beta^{2}\right)\left(X+2 Y \beta+Z \beta^{2}\right),
$$

with

$$
\begin{array}{ll}
X=\left\|b_{n} \boldsymbol{\nabla} \chi_{n}\right\|_{D}^{2}+\delta_{n}^{2}\left\|b_{n}\right\|_{D}^{2}, & A=F^{S}\left(\boldsymbol{W}_{s, n}\right)+F_{n}^{D}\left(\boldsymbol{W}_{s, n}, \chi_{n}\right), \\
Y=\operatorname{Re}\left\langle b_{n} \boldsymbol{\nabla} \chi_{n}, b_{n} \boldsymbol{\nabla} d_{n}\right\rangle_{D}, & B=\sum_{s}\left\|d_{n} \boldsymbol{E}_{s, n}\right\|_{D}^{2} / \sum_{s}\left\|\chi_{n-1} \boldsymbol{E}_{s}^{\text {inc }}\right\|_{D}^{2}, \\
Z=\left\|b_{n} \boldsymbol{\nabla} d_{n}\right\|_{D}^{2} . &
\end{array}
$$

Then, differentiation with respect to $\beta$ yields a cubic equation with one real root and two complex conjugate roots. The real root is the desired minimizer 
$\beta_{n}$. The cost functional $F_{n}$ in (21) is a convex function of real $\beta$ and has one minimum for real $\beta$ because its second derivative with respect to $\beta$ is positive. The second derivative is obtained as $\partial^{2} \mathcal{F}_{n} / \partial \beta^{2}=12 B Z \beta^{2}+12 B Y \beta+2(B X+A Z)$. For $\beta=0$ this function is positive, and remains positive when the right-hand side has no real zeros. This is the case if $Y^{2}<2 / 3\left(X Z+A Z^{2} / B\right)$. Since both $A$ and $B$ are functions of $\boldsymbol{w}_{s, n}$, we rather want a criterion in which only functions of contrast quantities occur. Further the term $A Z^{2} / B$ is always non-negative. Hence a sufficient condition that the second derivative is a positive function of $\beta$ is $Y^{2} \leq 2 / 3 X Z$ or

$$
\frac{\left[\operatorname{Re}\left\langle b_{n} \boldsymbol{\nabla} \chi_{n}, b_{n} \boldsymbol{\nabla} d_{n}\right\rangle_{D}\right]^{2}}{\left[\left\|b_{n} \boldsymbol{\nabla} \chi_{n}\right\|_{D}^{2}+\delta_{n}^{2}\left\|b_{n}\right\|_{D}^{2}\right]\left\|b_{n} \boldsymbol{\nabla} d_{n}\right\|_{D}^{2}} \leq \frac{2}{3} .
$$

But, by Cauchy-Schwarz, $\left[\operatorname{Re}\left\langle b_{n} \boldsymbol{\nabla} \chi_{n}, b_{n} \boldsymbol{\nabla} d_{n}\right\rangle_{D}\right]^{2} /\left(\left\|b_{n} \boldsymbol{\nabla} \chi_{n}\right\|_{D}^{2}\left\|b_{n} \boldsymbol{\nabla} d_{n}\right\|_{D}^{2}\right) \leq 1$. Thus

$$
\delta_{n}^{2} \geq\left\|b_{n} \nabla \chi_{n}\right\|_{D}^{2} /\left(2\left\|b_{n}\right\|_{D}^{2}\right)
$$

is a sufficient condition for the cost functional to be a convex function with one minimum. If the choice for the parameter $\delta_{n}^{2}$ of (11) is less than the righthand side of (24), we replace the value of $\delta_{n}^{2}$ by the right-hand side of (24) in which we take $b_{n}=\left[\int_{D} \mathrm{~d} v(\boldsymbol{x})\left(\left|\boldsymbol{\nabla} \chi_{n}\right|^{2}+\delta_{n-1}^{2}\right)\right]^{-1 / 2}$. We have refrained from using this value for $\delta_{n}^{2}$ in the whole iteration procedure because from our numerical observations it appears this value has a large variation at the beginning of the optimization procedure.

After we have obtained a new estimate $\chi_{n}^{R}$ for the contrast, we repeat again the updating of $\boldsymbol{W}_{s, n}$ (if the value of the cost functional is not small enough) starting with $\chi_{n-1}=\chi_{n-1}^{R}$ of the previous iteration.

\section{Numerical Examples}

\subsection{Two-Dimensional Inversion From TM Electromagnetic Data}

We first consider inversion from the 2D-TM polarization measurement. For this measurement there are experimental data available which have been measured using a circular microwave scanner operating at $2.33 \mathrm{GHz}$. The scanner consists of a $12.5 \mathrm{~cm}$ radius circular array of 64 water-immersed horn antennas [2]. Only the vertical componenent of the electric field parallel to the array axis (the $x_{3}$-axis) is measured. The measurement procedure records the total electric field values at the receiving antennas. If one antenna is transmitting, the fields are measured only with the 33 antennas located in front of the active source. The scattered fields are deduced from the total field by subtracting the incident field, measured in the absence of any targets. Further, the measured scattered fields have been calibrated so that a directed unit line source can be used as the model for the incident fields, viz., $E_{3, s}^{\text {inc }}(\boldsymbol{x})=-0.25 \omega \mu_{0} H_{0}^{(2)}\left(k_{\mathrm{b}}\left|\boldsymbol{x}-\boldsymbol{x}_{s}^{\mathrm{S}}\right|\right)$, where $\mu_{0}=4 \pi \times 10^{-7}$ is the permeability in vacuum. 

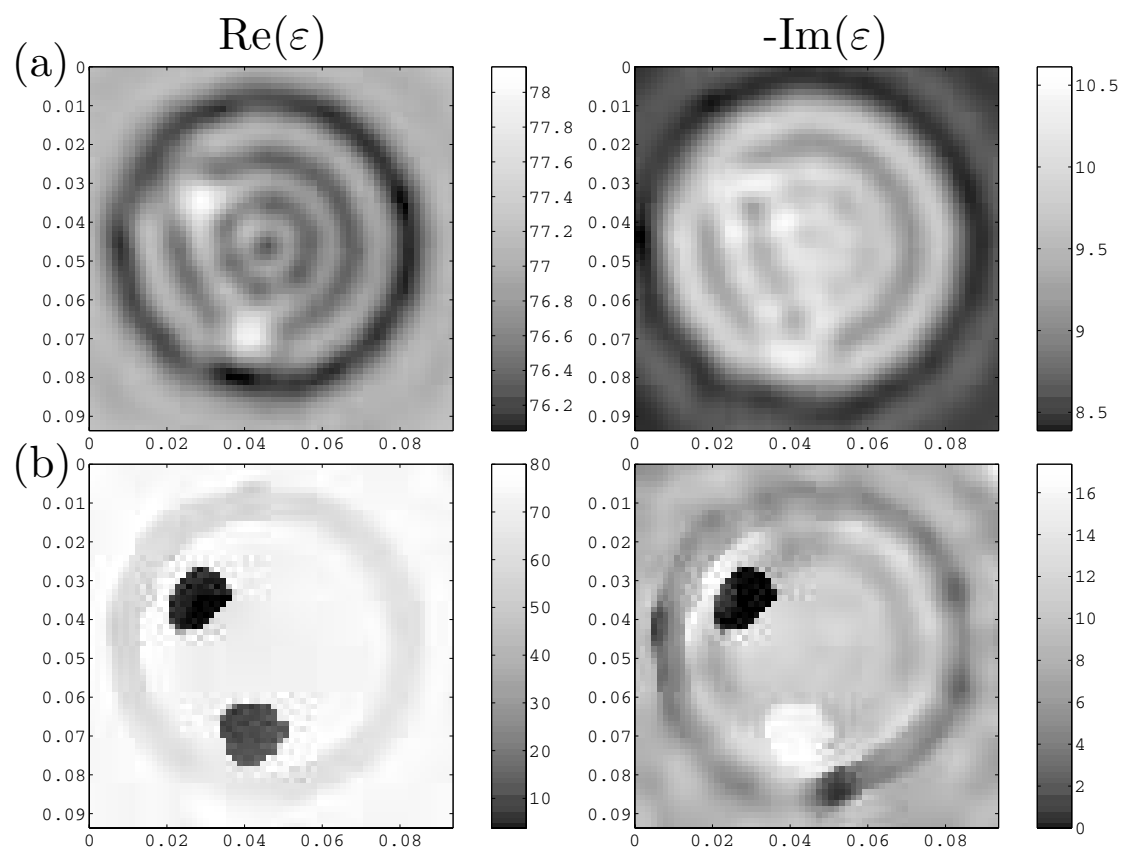

Fig. 1. Human arm phantom images: using back propagation (a) and MR-CSI (b).

In the inversion of experimental data we assumed that the unknown object is entirely located within a test domain $D$ with dimension of $6.4 \lambda$ by $6.4 \lambda$ where $\lambda$ is the wavelength in water. The permittivity of water is approximately $\epsilon_{\mathrm{b}}=$ $77.3-\mathrm{j} 8.66$ at frequency $f=2.33 \mathrm{GHz}$. Hence, the wavelength $\lambda=14.6 \mathrm{~mm}$. The discrete form of the algorithm is obtained by dividing the test domain $D$ into 64 by 64 subsquares. The discrete spatial convolutions are efficiently computed using FFT routines. The lower and upper bounds of the reconstructed complex permittivity in the inversion algorithm are enforced as $0 \leq \operatorname{Re}[\varepsilon(\boldsymbol{x})] / \varepsilon_{0} \leq 80$ and $0 \leq-\operatorname{Im}[\varepsilon(\boldsymbol{x})] / \varepsilon_{0} \leq 20$.

The first experimental data were obtained from a human arm phantom. The external layer (supposed to model the skin) and bones of the human arm phantom were made with PVC with complex permittivity $2.73-\mathrm{j} 0.01$ and the muscle was 54.5-j 17.2. We show first the results obtained from the initial estimates (back propagation). These results, approximately identical to those using the spectral diffraction tomography technique, are given in the top-plots of Fig. 1a. The results of the MR-CSI method after 1024 iterations are given in the bottomplots of Fig. 1b. Although the total number of iterations is large, the total computation time is very limited. Note that we do not solve any forward problem at each iteration of the algorithm. One iteration of the MR-CSI method takes approximately 8 seconds on a personal computer with $600 \mathrm{MHz}$ Pentium III processor. After 1024 iterations the normalized data error $F^{S}$ is already reduced to $6.33 \%$, and adding more iterations does not change the result. From the results, 

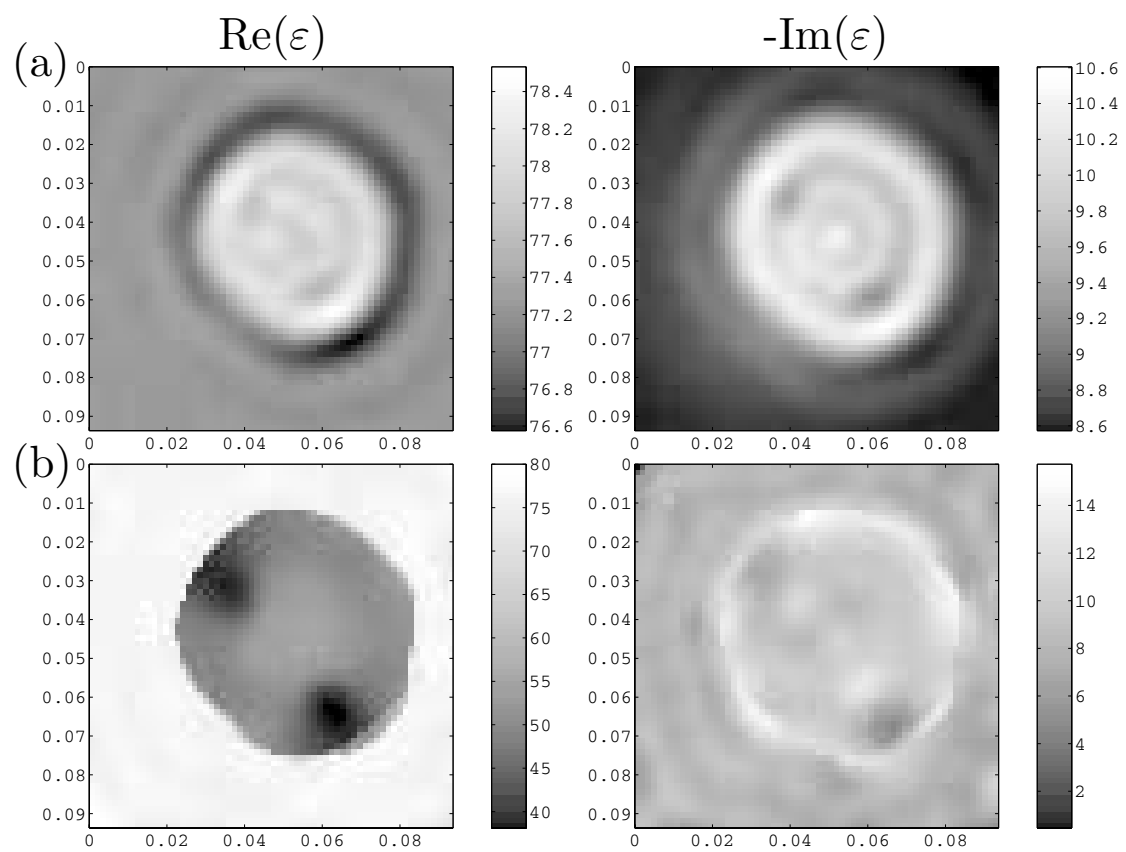

Fig. 2. Human forearm images: using back propagation (a) and MR-CSI method (b).

we observe that the bones are clear and sharp. The only drawback is that the reconstructed imaginary part of the complex permittivity for one of the bones is completely wrong. This can be caused by the presence of the noise in the experimental data.

For the second experiment we consider data that were taken from a human forearm. The back propagation results are given in the top-plots of Fig. 2a. The results of the MR-CSI method after 1024 iterations are given in Fig. 2b. After 1024 iterations the value of $F^{S}$ is already reduced to $4.10 \%$, and adding more iterations does not change the result. The reconstructed images show the positions of the two bones and the correct value of the muscle (approximately 54.5-i 17.2). Conversely, due to the water and tissue attenuation and the reduced dynamic range of the available data, the complex permittivity values of the bones are higher than the real ones (it should approximately be 5.5-i 0.59 at the present frequency of operation).

\subsection{Three-Dimensional Inversion from Vectorial Data}

As a test case for our full-vectorial 3D inversion algorithm we use the 3D neck model which is immersed in water with permittivity $\varepsilon_{\mathrm{b}}=78-\mathrm{j} 3.6$ at $1 \mathrm{GHz}$. The original profile of this neck model is given in Fig. 3a. The neck model consists of fat tissue with permittivity $28-\mathrm{j} 13.5$, cartilage with $25-\mathrm{j} 10.78$, veins/arteries with $63-\mathrm{j} 20$, bone with $6.4-\mathrm{j} 2.16$, trachea 1 , marrow $5.5-\mathrm{j} 0.59$, 

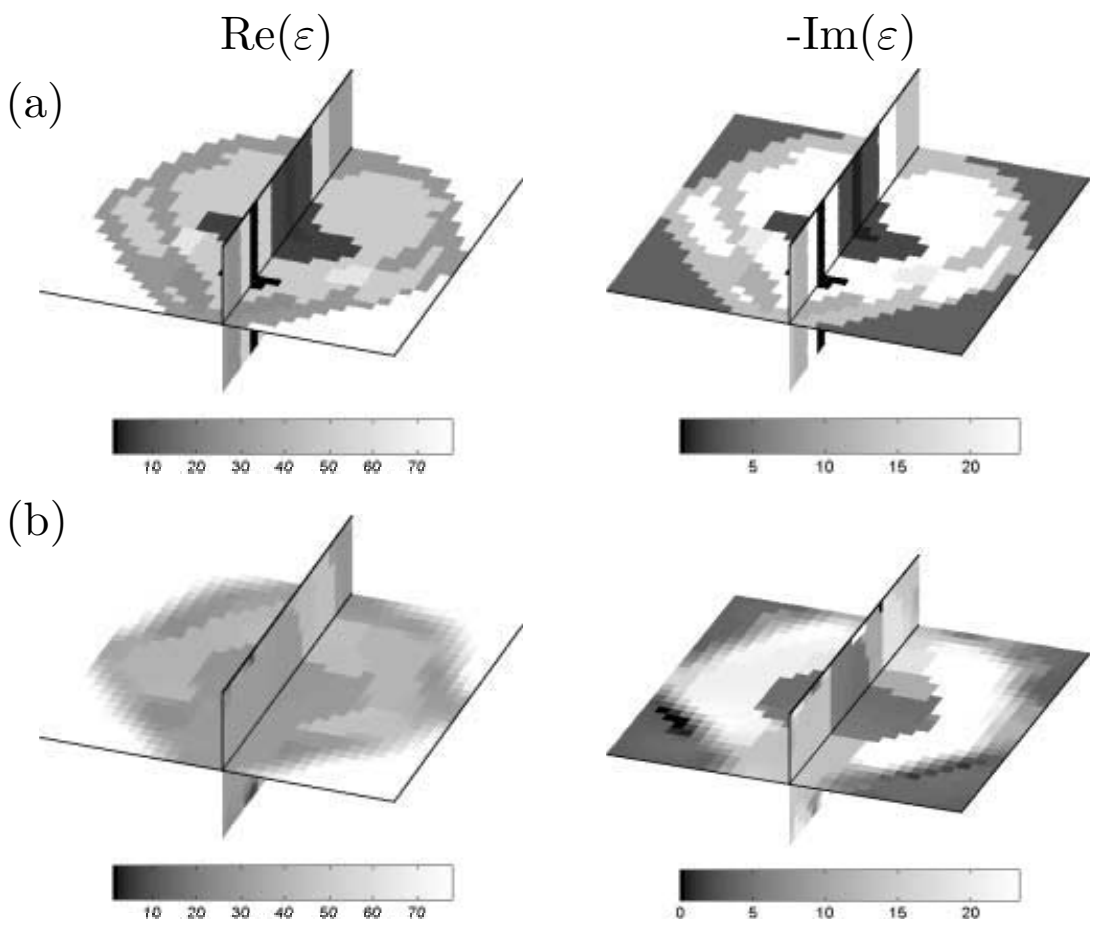

Fig. 3. Neck model: original 3D images (a) and reconstructed $3 \mathrm{D}$ images (b).

and muscle with $50-\mathrm{j} 23.37$. The size of the domain $D$ in Fig. 3a is $3.1 \lambda$ by $3.1 \lambda$ by $1.55 \lambda$ where $\lambda=1 / 30$ is the wavelength in water. The measurement setup is similar to the one used by Bulyshev et al. [3]. We have three rings containing 30 transmitter antennas and six rings containing 90 receiver antennas. All receivers operate simultaneously while the transmitters operate one after another. Thus, in total we have 2700 data points. The receiver records all the components of the scattered electric field $\boldsymbol{E}_{s}^{\text {sct }}$. The transmitter is modeled using a point magnetic dipole directed in the $x_{3}$-direction, viz., $\boldsymbol{E}_{s}^{\mathrm{inc}}(\boldsymbol{x})=\mathrm{j} \omega \mu_{0} \exp \left(-\mathrm{j} k_{\mathrm{b}}\left|\boldsymbol{x}-\boldsymbol{x}_{s}^{\mathrm{S}}\right|\right) / 4 \pi \mid \boldsymbol{x}-$ $\left.\boldsymbol{x}_{s}^{\mathrm{S}}\right|^{3}\left(-\mathrm{j} k_{\mathrm{b}}\left|\boldsymbol{x}-\boldsymbol{x}_{s}^{\mathrm{S}}\right|-1\right)\left[\left(x_{2}-x_{2, s}^{\mathrm{S}}\right) \boldsymbol{i}_{1}-\left(x_{1}-x_{1, s}^{\mathrm{S}}\right) \boldsymbol{i}_{2}\right]$. The vertical positions of the transmitter rings are $x_{3}^{\mathrm{S}}= \pm 0.35 \lambda$ and 0 , and of the receiver rings are $x_{3}^{\mathrm{S}}= \pm \lambda$, $\pm 0.5 \lambda$ and $\pm 0.25 \lambda$.

The original profile is given in Fig. 3a. These plots are the volume slices at $x_{1}=0$ and $x_{3}=0$. After generation of synthetic data a $5 \%$ random additive white noise is added. In the inversion we discretize the test domain $D$ into 31 by 31 by 15 cells, hence, the total number of unknowns is equal to 14415 . The reconstruction results after 1024 iterations are given in Fig. 3b. Note that one iteration takes approximately 22 seconds on a personal computer with a $600 \mathrm{MHz}$ Pentium III processor. We observe that the results are quite satisfactory in spite of the use of very limited data. 


\section{Conclusions}

The presented results for biomedical data using a 2D-TM polarization measurement and 3D vectorial data show that the contrast source inversion method using multiplicative regularization leads to an effective inversion technique. The algorithm is fully iterative and does not solve any forward problem in each iteration. This makes the method suitable for large scale computations. Furthermore, the artificial tuning process with a weighting parameter of the regularization to obtain the "cosmetically best" results seems superfluous.

\section{References}

1. Bolomey, J., Pichot, C.: Some applications of diffraction tomography to electromagnetics - the particular case of microwaves in: Inverse Problems in Scattering and Imaging, M. Bertero and E.R. Pike, Eds., London, U.K.: Adam Hilger (1982) 319-344

2. Broquetas, A., Romeu, J., Rius, J.M., Elias-Fuste, A.R., Cardama, A., Jofre, L.: Cylindrical geometry: A further step in active microwave tomography. IEEE Trans. Microwave Theory Tech. 39 (1991) 836-844

3. Bulyshev, A.E., Souvorov, A.E., Semenov, S.Y., Svenson, R.H., Nazarov, A.G., Sizov, Y.E., Tatsis, G.P.: Three-dimensional microwave tomography. Theory and computer experiments in scalar approximation. Inverse Probl. 16 (2000) 863-875

4. Chew, W.C., Wang, Y.M.: An iterative solution of two-dimensional electromagnetic inverse scattering problem. Int. J. Imag. Syst. Technol. 1 (1989) 100-108

5. Chew, W.C., Wang, Y.M.: Reconstruction of 2D permittivity distribution using the distorted Born iterative method. IEEE Trans. Med. Imag. 9 (1990) 218-225

6. Franchois A., Pichot, C.: Microwave imaging - complex permittivity reconstruction with a Levenberg-Marquadt method. IEEE Trans. Microwave Theory Tech. 46 (1997) 133-141

7. Harada, H., Wall, D., Takenaka, T., Tanaka, T.: Conjugate gradient method applied to inverse scattering problem. IEEE Trans. Antennas Propagat. 43 (1995) $784-792$

8. Joachimowicz, N., Mallorqui, J.J., Bolomey, J.Ch., Broquetas, A.: Convergence and stability assessment of Newton-Kantorovich reconstruction algorithms for microwave tomography. IEEE Trans. Med. Imag. 17 (1998) 562-569

9. Kleinman, R.E., van den Berg, P.M.: An extended range modified gradient technique for profile inversion, Radio Sci. 28 (1993), 877-884

10. Meaney, P.M., Paulsen, K.D., Hartov, A., Crane, R.K.: Microwave imaging for tissue assessment: Initial evaluation in multitarget tissue equivalent phantoms. IEEE Trans. Biomed. Enq. 43 (1996) 878-890

11. Souvorov, A.E., Bulyshev, A.E., Semenov, S.Y., Svenson, R.H., Nazarov, A.G., Sizov, Y.E., Tatsis, G.P.: Microwave tomography: A two-dimensional Newton iterative scheme. IEEE Trans. Microwave Theory Tech. 46 (1998) 1654-1659

12. van den Berg, P.M., Kleinman, R.E.: A contrast source inversion method. Inverse Probl. 13 (1997) 1607-1620

13. van den Berg, P.M., van Broekhoven, A.L., Abubakar, A.: Extended contrast source inversion. Inverse Probl. 15 (1999) 1325-1344

14. van den Berg P.M., Abubakar, A.: Contrast source inversion method: State of art. Prog. in Electromag. Research 34 (2001) 189-218 